\title{
Avaliação dos danos de Oebalus spp. em genótipos de arroz irrigado(1)
}

\author{
Evane Ferreira( ${ }^{(2)}$, Noris Regina de Almeida Vieira(2) e Paulo Hideo Nakano Rangel(2)
}

\begin{abstract}
Resumo - O objetivo deste trabalho foi avaliar a reação de genótipos de arroz irrigado ao dano causado por percevejos adultos (Oebalus spp., Hemiptera: Pentatomidae), confinados nas panículas, durante os períodos de desenvolvimento e maturação das espiguetas. Dez genótipos foram comparados pelas perdas quantitativas, qualitativas e totais provocadas por $O$. poecilus, no primeiro e segundo experimentos de campo, respectivamente. Paralelamente, cinco genótipos do primeiro experimento foram comparados quanto às perdas quantitativas causadas por $O$. ypsilongriseus. Ocorreu reação significativamente diferente entre os genótipos com relação às duas espécies de percevejo. O. poecilus causou uma perda geral média, nos dois experimentos, de 52,7\%. A porcentagem média de perda total foi maior em Metica 1 e CNA 7545, e menor, na linhagem CNA 8033.
\end{abstract}

Termos para indexação: Oryza sativa, espigueta, perda, inseto.

\section{Evaluation of damages caused by Oebalus spp. in irrigated rice genotypes}

\begin{abstract}
The objective of this study was to evaluate the reaction of irrigated rice genotypes to the damage caused by adult paddy bugs (Oebalus spp., Hemiptera: Pentatomidae) during spikelet development and maturation. Quantitative and qualitative losses, as well as total loss, caused by $O$. poecilus were compared among ten genotypes in the first and second field experiments, respectively. At the same time, five genotypes, in the first experiment, were also compared to damage caused by O. ypsilongriseus. Significant differences were observed among the evaluated genotypes in relation to reaction to insect species. For both experiments, the overall average loss caused by O. poecilus was $52.7 \%$. The average total loss was greater for Metica 1 and CNA 7545 and smaller for CNA 8033.
\end{abstract}

Index terms: Oryza sativa, spikelets, losses, insects.

\section{Introdução}

Os percevejos Oebalus poecilus (Dallas, 1851) e Oebalus ypsilongriseus (De Geer, 1773) (Hemiptera: Pentatomidae) incluem-se entre os principais insetos orizívoros no Brasil, tanto no ecossistema de várzeas como no de terras altas (Costa, 1958; Ferreira \& Martins, 1984; Link et al., 1987, 1989; Ferreira, 1998; Vieira et al., 1999). Os insetos localizam-se principalmente nas panículas, durante o período de desenvolvimento das espiguetas, nas quais, pela alimentação direta, provocam reduções quantitativas e qualitativas (Ferreira \& Martins, 1985; Martins et al., 1989; Oliveira \& Kempf, 1989; Ferreira, 1998; Vieira et al., 1999). Além disso, O. poecilus é vetor de fungos,

\footnotetext{
(1) Aceito para publicação em 15 de janeiro de 2002 .

(2) Embrapa-Centro Nacional de Pesquisa de Arroz e Feijão, Caixa Postal 179, CEP 75375-000 Santo Antônio de Goiás, GO. E-mail: evane@cnpaf.embrapa.br, noris@cnpaf.embrapa.br, phrangel@cnpaf.embrapa.br
}

que, aliados à sua atividade alimentar, contribuem para aumentar a incidência de manchas nas espiguetas (Kennard, 1966; Antoniolli, 1988; Vieira et al., 1999). O. poecilus e O. ypsilongriseus têm sido encontrados em proporções semelhantes em lavouras de arroz irrigado no Rio Grande do Sul (Costa, 1958; Link et al., 1989). Danos de O. poecilus em arroz irrigado foram relatados por Kennard (1966), Rai (1974), Link et al. (1987) e Martins et al. (1989). Não existem informações dessa natureza sobre O. ypsilongriseus.

Relações entre populações de plantas de arroz e perdas quantitativas, qualitativas, ou ambas, decorrentes de níveis de infestação de Oebalus spp., visando adequar medidas de controle ou para avaliação de resistência de plantas hospedeiras, têm sido estudadas em experimentos de campo, utilizando gaiolas teladas de vários tamanhos (Swanson \& Newsom, 1962; Kennard, 1966; Latson et al., 1976; Bowling, 1979; Robinson et al., 1981; Heinrichs et al., 1985; Link et al., 1987; Martins et al., 1989). Depen- 
dendo das características de consumo e de mercado de arroz, quatro exemplares de O. poecilus $/ \mathrm{m}^{2}$ de cultura são suficientes para definir a necessidade de controle (Weber, 1989).

Nilakhe (1976) estudou a resistência de cultivares e linhagens de arroz a $O$. pugnax, pela observação de perdas quantitativas e qualitativas, separadamente. No primeiro caso, utilizou como parâmetro de comparação a diferença entre a massa média por espigueta obtida para a testemunha e a parte infestada e, no segundo, a porcentagem de espiguetas manchadas. Robinson et al. (1981), também com o objetivo de avaliar a resistência de linhagens de arroz ao dano do mesmo inseto, utilizaram diversas variáveis comparativas e obtiveram reações diferenciadas das linhagens. Estes autores concluíram que o melhor critério, e o mais preciso, foi o da porcentagem total de dano, obtida pela soma do número de espiguetas vazias, de grãos parcialmente desenvolvidos e de grãos completamente desenvolvidos e manchados, dividindo-se esse valor pelo total de espiguetas (cheias e vazias) da amostra e multiplicando por 100 .

O objetivo deste trabalho foi avaliar a reação de genótipos de arroz irrigado ao dano de $O$. poecilus, e obter informações preliminares sobre o dano de O. ypsilongriseus, comparativamente ao de O. poecilus, sobre alguns genótipos de arroz irrigado.

\section{Material e Métodos}

Dois experimentos foram realizados: um, em 1996/97, e outro, em 1997/98, na Fazenda Palmital da Embrapa-Centro Nacional de Pesquisa de Arroz e Feijão, em Goianira, GO, utilizando delineamento experimental de blocos ao acaso, com parcelas subdivididas e quatro repetições. A adubação, idêntica nos dois anos, consistiu na aplicação de $400 \mathrm{~kg} / \mathrm{ha}$ da fórmula 4-30-16, no sulco de plantio, e de $150 \mathrm{~kg} /$ ha de sulfato de amônio, em cobertura, aos 50 dias após a semeadura.

A parcela experimental consistiu de uma fileira de plantas, com 2,0 e 3,5 m de comprimento, no primeiro e segundo experimento, respectivamente, com espaçamento, entre elas, de $0,8 \mathrm{~m}$, para facilitar as atividades de engaiolar e tutorar as panículas a serem infestadas. As subparcelas foram constituídas de quatro gaiolas com panículas de arroz, a saber: duas gaiolas infestadas, e duas testemunhas sem infestação. As gaiolas consistiram de estruturas de arame $(18 \mathrm{~cm}$ de diâmetro e $20 \mathrm{~cm}$ de altura) revestidas por mangas de filó de náilon $(20$ × $30 \mathrm{~cm})$. A manga de cada gaiola foi amarrada na extremidade superior e na inferior, juntamente com os colmos providos de panículas a um suporte de bambu ajustado ao longo da parte interna da gaiola.

No primeiro experimento, em cada gaiola foram isoladas duas panículas e quatro insetos. No segundo, manteve-se a mesma proporção inseto/panícula, contudo, para facilitar o isolamento e o monitoramento dos insetos confinados, decidiu-se deixar uma panícula e dois insetos. As infestações foram feitas com insetos adultos, criados sobre panículas de arroz, em casa telada. Após a infestação, foram realizadas vistorias a intervalos de dois a três dias para eliminar posturas e repor os percevejos mortos. Esse procedimento foi adotado até a colheita, realizada quando as panículas estavam completamente maduras.

No primeiro experimento, a avaliação do dano causado por $O$. poecilus foi realizada em dez genótipos (Javaé, Jequitibá, Metica 1, Formoso, CNA 8003, CNA 8033, CNA 7204, CNA 7857, CNA 7545 e CNA 7556). Dada a baixa disponibilidade de $O$. ypsilongriseus, somente cinco deles (CNA 7545, CNA 8003, CNA 8033, Javaé e Jequitibá) foram avaliados separadamente em relação a $O$. ypsilongriseus. No segundo experimento, por essa mesma razão, foi medido somente o dano de $O$. poecilus, tendo sido acrescentadas quatro linhagens (CNA 6343, CNA 8467, CNA 8470 e CNA 8487) aos dez genótipos utilizados no primeiro experimento.

Os experimentos foram instalados na época normal de plantio. No primeiro experimento, os genótipos foram semeados simultaneamente, em três datas (13 e 26/11 e 9/12/96). No segundo, a semeadura também foi feita em três datas (11, 20 e 25/11/97), mas, nesse caso, de acordo com o ciclo dos genótipos, sendo os de ciclo mais longo plantados na primeira data. Esse procedimento foi adotado para garantir a disponibilidade de panículas de idades não muito diferentes no momento da infestação. Nos dois experimentos, as panículas foram isoladas quando as espiguetas do ápice apresentavam o endosperma em estágio leitoso.

As panículas foram colhidas com as respectivas gaiolas, e a produção de grãos de cada gaiola foi registrada separadamente. Depois da secagem, em estufa a $50^{\circ} \mathrm{C} \mathrm{du}-$ rante três dias, as espiguetas foram separadas em vazias e com grãos, para contagem e pesagem. De cada genótipo, as espiguetas com grãos obtidas em cada repetição foram reunidas, conforme o tratamento, em infestadas e não-infestadas, formando quatro repetições com maior número de espiguetas. De cada repetição, foram amostradas 50 espiguetas, por genótipo, para avaliar a atividade alimentar dos insetos e seus efeitos sobre o poder germinativo das espiguetas. Foram determinados, também, a massa e o número total de espiguetas por panícula, a porcentagem de 
espiguetas vazias (número de espiguetas vazias/número total de espiguetas x 100), e a porcentagem de perda de massa de espiguetas das panículas infestadas em relação às não-infestadas, utilizando o método descrito em Heinrichs et al. (1985).

A atividade dos insetos foi avaliada por meio das seguintes determinações: número de bainhas de estilete, ou sinais de alimentação, por espigueta (Bowling, 1979); porcentagem de espiguetas com bainhas; porcentagem de perda de germinação, calculada com base na subtração do poder germinativo dos tratamentos infestados do valor obtido na testemunha sem infestação. A presença de bainhas de estilete nas espiguetas foi detectada utilizando o método da fucsina ácida, e o poder germinativo das sementes foi determinado pelo teste de germinação.

$\mathrm{Na}$ determinação da porcentagem de perda de massa de espiguetas, considerou-se as espiguetas vazias e as com grãos, constituindo a perda quantitativa.

A porcentagem total de perda de rendimento (PPT) provocada por $O$. poecilus nos genótipos de ambos experimentos foi obtida pela fórmula:

$\mathrm{PPT}=\mathrm{PPM}+[(100-\mathrm{PPM}) \mathrm{x}$ PPG/100]

em que: PPM é a porcentagem de perda de massa; e PPG é a porcentagem de perda de germinação.
Os dados foram submetidos à análise de variância simples. Os dados dos cinco genótipos comuns aos dois insetos, no primeiro experimento, e os dos 10 genótipos comuns entre o primeiro e segundo experimentos foram analisados conjuntamente (SAS Institute, 1990). As porcentagens foram analisadas sem e com transformação em arc sen $(\mathrm{p} / 100)^{0,5}$, e as médias, comparadas pelo teste de Tukey a $5 \%$ de probabilidade.

\section{Resultados e Discussão}

O. ypsilongriseus causou aumento significativo da porcentagem de espiguetas vazias, indistintamente, sobre os cinco genótipos avaliados. Entretanto, os genótipos apresentaram reação diferenciada quanto à porcentagem de perda de massa, a qual foi significativamente menor em Javaé e maior em CNA 8033 e Jequitibá (Tabela 1).

A análise conjunta das variáveis comuns às duas espécies de percevejos não apresentou interações significativas dos genótipos com a presença ou ausência das espécies de percevejos considerados, nem existiu diferença entre genótipos quanto à porcentagem de perda de massa (Tabela 2). Contudo, notou-se

Tabela 1. Variáveis relacionadas às panículas de arroz irrigado, infestadas e não-infestadas com Oebalus ypsilongriseus no primeiro experimento. Goianira, GO, 1996/97(1).

\begin{tabular}{|c|c|c|c|c|}
\hline $\begin{array}{l}\text { Cultivares e } \\
\text { linhagens }\end{array}$ & $\begin{array}{c}\text { Massa de espiguetas/panícula } \\
(\mathrm{g})\end{array}$ & $\begin{array}{l}\text { Espiguetas/panícula } \\
\left(\mathrm{n}^{\mathrm{o}}\right)\end{array}$ & $\begin{array}{c}\text { Espiguetas vazias } \\
(\%)^{(2)}\end{array}$ & $\begin{array}{l}\text { Perda de massa das espiguetas } \\
\qquad(\%)^{(2)}\end{array}$ \\
\hline CNA 8003 & $1,852 \mathrm{a}$ & $113,0 \mathrm{ab}$ & $33,6 a$ & $24,2 \mathrm{ab}$ \\
\hline Javaé & $1,522 \mathrm{a}$ & $90,6 b$ & $21,2 \mathrm{a}$ & $20,0 \mathrm{~b}$ \\
\hline CNA 8033 & $1,793 a$ & $98,0 \mathrm{ab}$ & $19,1 \mathrm{a}$ & $31,9 \mathrm{a}$ \\
\hline CNA 7545 & $1,775 \mathrm{a}$ & $106,4 \mathrm{ab}$ & $22,4 \mathrm{a}$ & $30,0 \mathrm{ab}$ \\
\hline Jequitibá & $2,009 \mathrm{a}$ & $120,0 \mathrm{a}$ & $32,2 \mathrm{a}$ & $44,4 \mathrm{a}$ \\
\hline Médias & 1,790 & 105,6 & 25,7 & 30,1 \\
\hline CV $(\%)$ & 33,4 & 24,9 & 38,4 & 42,0 \\
\hline Com infestação & $1,439 b$ & $104,0 \mathrm{a}$ & $35,8 \mathrm{a}$ & - \\
\hline Sem infestação & $2,142 \mathrm{a}$ & $107,2 \mathrm{a}$ & $15,6 \mathrm{~b}$ & - \\
\hline
\end{tabular}

${ }^{(1)}$ Médias seguidas pela mesma letra, nas colunas, não diferem entre si pelo teste de Tukey a $5 \%$ de probabilidade. ${ }^{(2)}$ Analisadas após transformação em $\operatorname{arcsen}(\mathrm{p} / 100)^{0,5}$.

Tabela 2. Variáveis relacionadas à infestação de panículas de arroz irrigado com Oebalus ypsilongriseus e O. poecilus, no primeiro experimento. Goianira, GO, 1996/97(1).

\begin{tabular}{|c|c|c|c|c|}
\hline $\begin{array}{l}\text { Cultivares e } \\
\text { linhagens } \\
\end{array}$ & $\begin{array}{c}\text { Espiguetas com bainha } \\
(\%)^{(2)}\end{array}$ & Bainhas por espigueta & $\begin{array}{c}\text { Espiguetas vazias } \\
(\%)^{(2)}\end{array}$ & $\begin{array}{l}\text { Perda de massa de espiguetas } \\
(\%)^{(2)} \\
\end{array}$ \\
\hline CNA 8003 & $65,9 \mathrm{ab}$ & $2,5 \mathrm{ab}$ & $35,9 \mathrm{a}$ & $31,8 \mathrm{a}$ \\
\hline Javaé & $50,1 b$ & $2,2 b$ & $27,5 \mathrm{ab}$ & $30,7 \mathrm{a}$ \\
\hline CNA 8033 & $71,8 \mathrm{a}$ & $2,9 \mathrm{ab}$ & $23,1 b$ & $30,3 a$ \\
\hline CNA 7545 & $73,0 \mathrm{a}$ & $3,7 \mathrm{a}$ & $26,0 \mathrm{ab}$ & $37,8 \mathrm{a}$ \\
\hline Jequitibá & $57,9 \mathrm{~b}$ & $2,2 \mathrm{~b}$ & $31,1 \mathrm{a}$ & $40,3 \mathrm{a}$ \\
\hline Médias & 64,9 & 2,7 & 28,7 & 34,2 \\
\hline $\mathrm{CV}(\%)$ & 18,7 & 59,2 & 43,0 & 42,2 \\
\hline Com O. ypsilongriseus & $59,1 \mathrm{~b}$ & $1,8 \mathrm{~b}$ & $25,2 \mathrm{~b}$ & $30,1 \mathrm{a}$ \\
\hline Com O.poecilus & $70,8 \mathrm{a}$ & $3,5 \mathrm{a}$ & $31,7 \mathrm{a}$ & $38,3 \mathrm{a}$ \\
\hline
\end{tabular}

${ }^{(1)}$ Médias seguidas pela mesma letra, nas colunas, não diferem entre si pelo teste de Tukey a $5 \%$ de probabilidade. ${ }^{(2)}$ Analisadas após tranformação em $\operatorname{arcsen}(\mathrm{p} / 100)^{0,5}$. 
uma tendência de $O$. ypsilongriseus causar menor porcentagem de perda de massa nas espiguetas que O. poecilus, em conseqüência de uma menor atividade, conforme demonstrado pela menor porcentagem de espiguetas vazias, menor porcentagem de espiguetas com bainha de estilete e menor número de bainhas de estilete por espigueta (Tabela 2).

A análise conjunta das perdas de massa (quantitativas), de germinação (qualitativas) e total (quantitativas + qualitativas) mostrou interações significativas entre genótipo e experimento (Tabelas 3, 4 e 5), o que indica, em cada caso, que os genótipos se comportaram de maneira diferente em relação aos experimentos. $\mathrm{Na}$ análise conjunta, todos os genótipos comportaram-se igualmente com relação à perda de massa, o mesmo acontecendo no primeiro experimento (Tabela 3). No segundo experimento, ocorreram diferenças significativas nas perdas de massa dos genótipos, destacando-se CNA 7857 e Metica 1 com maior perda em relação à Javaé, superando, inclusive, as perdas observadas no primeiro experimento. A média de perda de massa foi significativamente maior no primeiro experimento. Isso provavelmente aconteceu pela ocorrência de condições meteorológicas favoráveis à atividade do inseto, e, ao mesmo tempo, desfavoráveis ao desenvolvimento da fase reprodutiva das plantas da maioria dos genótipos no primeiro experimento (Costa, 1958; Vieira et al., 1999).

Tabela 3. Perda quantitativa dos genótipos de arroz irrigado causada pela infestação de adultos de Oebalus poecilus, em número de quatro exemplares por duas panículas no primeiro experimento, e dois exemplares por uma panícula no segundo experimento. Goianira, GO, 1996/98(1).

\begin{tabular}{lccr}
\hline Genótipos & \multicolumn{3}{c}{ Porcentagem de perda de massa } \\
\cline { 2 - 4 } & Experimento 1 & Experimento 2 & Conjunta \\
\hline Formoso & $30,5 \mathrm{Aa}$ & $13,7 \mathrm{Bab}$ & $24,1 \mathrm{a}$ \\
CNA 8003 & $39,4 \mathrm{Aa}$ & $25,9 \mathrm{Bab}$ & $32,7 \mathrm{a}$ \\
Javaé & $41,3 \mathrm{Aa}$ & $6,8 \mathrm{Bb}$ & $24,1 \mathrm{a}$ \\
CNA 8033 & $29,0 \mathrm{Aa}$ & $15,4 \mathrm{Bab}$ & $22,2 \mathrm{a}$ \\
CNA 7204 & $44,7 \mathrm{Aa}$ & $18,5 \mathrm{Bab}$ & $31,6 \mathrm{a}$ \\
CNA 7857 & $17,8 \mathrm{Ba}$ & $37,5 \mathrm{Aa}$ & $27,7 \mathrm{a}$ \\
Metica 1 & $28,1 \mathrm{Ba}$ & $34,8 \mathrm{Aa}$ & $31,5 \mathrm{a}$ \\
CNA 7545 & $45,6 \mathrm{Aa}$ & $30,2 \mathrm{Bab}$ & $37,9 \mathrm{a}$ \\
CNA 7556 & $50,1 \mathrm{Aa}$ & $28,5 \mathrm{Bab}$ & $39,3 \mathrm{a}$ \\
Jequitibá & $36,3 \mathrm{Aa}$ & $21,5 \mathrm{Bab}$ & $28,9 \mathrm{a}$ \\
\hline Médias & $36,3 \mathrm{~A}$ & $23,3 \mathrm{~B}$ & 29,8 \\
CV (\%) & 27,8 & 31,2 & 28,7 \\
\hline
\end{tabular}

${ }^{(1)}$ Médias seguidas pela mesma letra, maiúsculas nas linhas e minúsculas nas colunas, não diferem entre si pelo teste de Tukey a 5\% de probabilidade. ${ }^{(2)}$ Analisadas com transformação em $\operatorname{arc} \operatorname{sen}(\mathrm{p} / 100)^{0,5}$.
A análise conjunta das perdas qualitativas apresentou diferenças significativas entre os genótipos, destacando-se a cultivar Metica 1, com maior perda em relação à cultivar Jequitibá e à linhagem CNA 7556 (Tabela 4). Não houve diferença na perda média observada entre o primeiro e segundo experimentos. Contudo, foram observadas diferenças significativas nas perdas qualitativas nos genótipos dentro de cada experimento, mostrando-se alguns mais afeta-

Tabela 4. Perda qualitativa dos genótipos de arroz irrigado, provenientes de panículas infestadas com adultos de Oebalus poecilus, em número de quatro exemplares por duas panículas no primeiro experimento, e dois exemplares por uma panícula no segundo experimento. Goianira, GO, 1996/98(1).

\begin{tabular}{lccc}
\hline Genótipos & \multicolumn{3}{c}{ Porcentagem de perda de poder germinativo ${ }^{(2)}$} \\
\cline { 2 - 4 } & Experimento 1 & Experimento 2 & Conjunta \\
\hline Formoso & $40,8 \mathrm{Aab}$ & $21,0 \mathrm{Bcde}$ & $30,9 \mathrm{ab}$ \\
CNA 8003 & 29,5Babc & $37,3 \mathrm{Aabc}$ & $33,4 \mathrm{ab}$ \\
Javaé & 46,3Aa & $19,3 \mathrm{Bde}$ & $32,8 \mathrm{ab}$ \\
CNA8033 & $45,5 \mathrm{Aa}$ & $17,3 \mathrm{Be}$ & $31,4 \mathrm{ab}$ \\
CNA 7204 & $38,8 \mathrm{Aab}$ & $36,3 \mathrm{Babcd}$ & $37,5 \mathrm{ab}$ \\
CNA 7857 & $21,8 \mathrm{Bbc}$ & $41,5 \mathrm{Aab}$ & $31,6 \mathrm{ab}$ \\
Metica 1 & $39,8 \mathrm{Bab}$ & $49,0 \mathrm{Aa}$ & $44,4 \mathrm{a}$ \\
CNA 7545 & 40,0Aab & $34,0 \mathrm{Babcd}$ & $37,0 \mathrm{ab}$ \\
CNA 7556 & $13,3 \mathrm{Bc}$ & $36,5 \mathrm{Aabcd}$ & $24,9 \mathrm{~b}$ \\
Jequitibá & $21,5 \mathrm{Bbc}$ & $30,0 \mathrm{Abcde}$ & $25,8 \mathrm{~b}$ \\
\hline Médias & $33,7 \mathrm{~A}$ & $32,2 \mathrm{~A}$ & 33,0 \\
CV (\%) & 16,4 & 12,9 & 15,9 \\
\hline
\end{tabular}

(1)Médias seguidas pela mesma letra, maiúsculas nas linhas e minúsculas nas colunas, não diferem entre si pelo teste de Tukey a 5\% de probabilidade. ${ }^{(2)}$ Analisadas com transformação em $\operatorname{arc} \operatorname{sen}(\mathrm{p} / 100)^{0,5}$.

Tabela 5. Perda total de rendimento (qualitativa e quantitativa) dos genótipos de arroz irrigado, provenientes de panículas infestadas com adultos de Oebalus poecilus, em número de quatro exemplares por duas panículas no primeiro experimento, e dois exemplares por uma panícula no segundo experimento. Goianira, GO, 1996/98 ${ }^{(1)}$.

\begin{tabular}{llcl}
\hline Genótipos & \multicolumn{3}{c}{ Porcentagem de perda total $^{(2)}$} \\
\cline { 2 - 4 } & Experimento 1 & Experimento 2 & Conjunta \\
\hline Formoso & $60,1 \mathrm{Aab}$ & $31,9 \mathrm{Bcd}$ & $46,0 \mathrm{ab}$ \\
CNA 8003 & $57,7 \mathrm{Aab}$ & $53,6 \mathrm{Bab}$ & $55,7 \mathrm{ab}$ \\
Javaé & $68,9 \mathrm{Aa}$ & $25,0 \mathrm{Bb}$ & $47,0 \mathrm{ab}$ \\
CNA 8033 & $60,5 \mathrm{Aab}$ & $30,0 \mathrm{Bcd}$ & $45,3 \mathrm{~b}$ \\
CNA 7204 & $67,6 \mathrm{Aa}$ & $47,9 \mathrm{Babc}$ & $57,7 \mathrm{ab}$ \\
CNA 7857 & 35,6Bb & $62,8 \mathrm{Aab}$ & $49,2 \mathrm{ab}$ \\
Metica 1 & $55,9 \mathrm{Bab}$ & $67,0 \mathrm{Aa}$ & $61,5 \mathrm{a}$ \\
CNA 7545 & $68,4 \mathrm{Aa}$ & $54,1 \mathrm{Bab}$ & $61,2 \mathrm{a}$ \\
CNA 7556 & $57,1 \mathrm{Aab}$ & $54,3 \mathrm{Bab}$ & $55,7 \mathrm{ab}$ \\
Jequitibá & $50,5 \mathrm{Aab}$ & $45,9 \mathrm{Bbc}$ & $48,2 \mathrm{ab}$ \\
\hline Média & $58,2 \mathrm{~A}$ & $47,3 \mathrm{~B}$ & 52,7 \\
CV (\%) & 13,2 & 10,9 & 12,1 \\
\hline
\end{tabular}

(1)Médias seguidas pela mesma letra, maiúsculas nas linhas e minúsculas nas colunas, não diferem entre si pelo teste de Tukey a 5\% de probabilidade. ${ }^{(2)}$ Analisadas com transformação em arc sen $(\mathrm{p} / 100)^{0,5}$. 
dos no primeiro experimento e menos no segundo experimento.

Tanto um critério de avaliação como o outro, isoladamente, não são suficientes para avaliar a reação de genótipos de arroz que, sob infestação de percevejos das panículas sofram menos perda de massa e ao mesmo tempo proporcionem um produto de boa qualidade para semente e para consumo alimentar (Tabelas 3 e 4). Para atingir resultados satisfatórios neste sentido, deve ser usada a porcentagem total de perda (Tabela 5), o que está de acordo com as considerações de Robinson et al. (1981). Ainda assim, o critério utilizado por estes autores pode não refletir o grau de severidade do dano na germinação, a qual varia de acordo com o ponto de alimentação do percevejo no endosperma (Swanson \& Newsom, 1962). A avaliação das perdas pelo poder germinativo contempla esse aspecto.

A análise conjunta da perda total de rendimento mostra reação diferenciada dos genótipos, destacando-se a cultivar Metica 1 e a linhagem CNA 7545 como os mais danificados em relação à linhagem CNA 8033, ficando os demais genótipos em situação intermediária (Tabela 5). A média da perda total do primeiro experimento foi significativamente maior do que a do segundo experimento, observando-se, contudo, comportamento diferenciado dos genótipos CNA 7857 e Metica 1, que, no segundo experimento tiveram perdas significativamente maiores do que no primeiro experimento. Uma decisão de controle com base na perda total avaliada deve ser muito mais rigorosa do que a baseada apenas na perda de massa. Desse modo, se o nível de controle econômico é " $n$ " insetos no início da emissão das panículas do arroz, com base na perda total, esse valor deverá ser reduzido aproximadamente à metade $(\mathrm{n} / 2)$, considerando a média geral de perda de rendimento dos genótipos.

\section{Conclusões}

1. Sobre os mesmos genótipos, Oebalus ypsilongriseus causa perda de massa de espigueta semelhante ao Oebalus poecilus, embora seja menos ativo.

2. Há diferença na reação dos genótipos de arroz irrigado com relação à perda total de espiguetas.

3. A perda total (quantitativa e qualitativa) de espiguetas é um critério que reflete o dano real do percevejo em genótipos de arroz.

\section{Referências}

ANTONIOLLI, Z. I. Natureza do "pecky rice" do arroz parbolizado no Rio Grande do Sul. 1988. 136 f. Dissertação (Mestrado) - Universidade Federal do Rio Grande do Sul, Porto Alegre.

BOWLING, C. C. The stylet sheath as an indicator of feeding activity of the rice stink bug. Journal of Economic Entomology, Lanham, v. 72, n. 2, p. 259-260, Apr. 1979.

COSTA, R. G. Alguns insetos e outros pequenos animais que danificam plantas cultivadas no Rio Grande do Sul. Porto Alegre: Secretaria de Estado dos Negócios da Agricultura, Indústria e Comércio, 1958. 296 p.

FERREIRA, E. Manual de identificação de pragas do arroz. Santo Antônio de Goiás: Embrapa-CNPAF, 1998. 110 p. (Documentos, 90).

FERREIRA, E.; MARTINS, J. F. da S. Insetos prejudiciais ao arroz no Brasil e seu controle. Goiânia: EmbrapaCNPAF, 1984. 67 p. (Documentos, 11).

FERREIRA, E.; MARTINS, J. F. da S. Insetos prejudiciais às panículas do arroz de sequeiro. Goiânia, Embrapa-CNPAF, 1985. 5 p. (Comunicado Técnico, 18).

HEINRICHS, E.; MEDRANO, F. G.; RAPUSAS, H. R. Genetic evaluation for insect resistance in rice. Los Baños: International Rice Research Institute, 1985. 356 p.

KENNARD, C. P. Effect of the paddy bug, O. poecilus on rice yield and quality in British Guyana. Lanham: FAO, 1966. p. 54-57. (Plant Protection Bulletin, 14).

LATSON, L. N.; TRAHAN, G. B.; RAO, S. V. R. Evaluation for resistance to rice insects. Louisiana State University Rice Experiment Station Annual Progress Report, Crowley, p. 125-144, 1976.

LINK, D.; COSTA, E. C.; MARCHEZAN, E. Avaliação preliminar de diferentes densidades de Oebalus poecilus (Dallas, 1851) sobre o rendimento do arroz. In: REUNIÃO DA CULTURA DO ARROZ IRRIGADO, 16., 1987, Balneário Camboriú. Anais... Florianópolis: Empasc, 1987. p. 229-232.

LINK, D.; COSTA, E. C.; TARRAGÓ, M. F. S. Ocorrência de percevejos pentatomídeos em lavouras de arroz na região central do Rio Grande do Sul. In: REUNIÃO DA CULTURA DO ARROZ IRRIGADO, 18., 1989, Porto 
Alegre. Anais... Porto Alegre: Instituto Rio-Grandense do Arroz, 1989. p. 346-353.

MARTINS, J. F. da S.; RIBEIRO, A. S.; TERRES, A. L. S. Danos causados pelo percevejo-do-grão ao arroz irrigado. In: REUNIÃO DA CULTURA DO ARROZ IRRIGADO, 18., 1989, Porto Alegre. Anais... Porto Alegre: Instituto Rio-Grandense do Arroz , 1989. p. 396-404.

NILAKHE, S. S. Rice lines screened for resistance to the rice stink bug. Journal of Economic Entomology, Lanham, v. 69, n. 6, p. 703-705, 1976.

OLIVEIRA, J. V. de; KEMPF, D. Avaliação de danos do arroz irrigado pelo percevejo-do-grão(Oebalus poecilus, Dallas, 1851). In: REUNIÃO DA CULTURA DO ARROZ IRRIGADO, 18., 1989, Porto Alegre. Anais... Porto Alegre: Instituto RioGrandense do Arroz, 1989. p. 405-409.

RAI, B. K. Losses caused by the paddy bug and "red rice" in Guyana. Lanham: FAO, 1974. p. 82-86. (Plant Protection Bulletin, 22).
ROBINSON, B. F.; GIFFORD, J. R.; TRAHAN, G. B. Evaluation of 32 uniform rice nursery lines for rice stink bug resistance. Louisiana State University Rice Experiment Station Annual Progress Report, Crowley, p. 278-285, 1981.

SAS INSTITUTE (Cary, Estados Unidos). SAS insight user's guide: version 6. Cary, 1990. $441 \mathrm{p}$

SWANSON, M. C.; NEWSOM, L. D. Effect of infestation by the rice stink bug, Oebalus pugnax, on yield and quality in rice. Journal of Economic Entomology, Lanham, v. 55, n. 6 , p. $877-879,1962$.

VIEIRA, N. R. de A.; SANTOS, A. B.; SANT'ANA, E. P. A cultura do arroz no Brasil. Santo Antônio de Goiás: Embrapa-CNPAF, 1999. 633 p.

WEBER, G. Desarrollo del manejo integrado de plagas del cultivo de arroz. Cali: Centro Internacional de Agricultura Tropical, 1989. 69 p. (Série 04.04). 